\title{
OPEN Identification of plaque ruptures using a novel discriminative model comprising biomarkers in patients with acute coronary syndrome
}

Hyungdon Kook ${ }^{1}$, Duck Hyun Jang ${ }^{1}$, Jong-Ho Kim ${ }^{1}$, Jae-Young Cho ${ }^{2}$, Hyung Joon Joo ${ }^{1}$, Sang-A Cho ${ }^{3}$, Jae Hyoung Park ${ }^{1}$, Soon Jun Hong ${ }^{1}$, Cheol Woong Yu ${ }^{1 \bowtie}$ \& Do-Sun Lim ${ }^{1 \bowtie}$

Soluble lectin-like oxidized low-density lipoprotein receptor-1 (sLOX-1), neutrophil gelatinaseassociated lipocalin (NGAL), and matrix metalloproteinase-9 (MMP-9) are inflammatory biomarkers involved in plaque destabilization resulting in acute coronary syndrome (ACS). This study aimed to investigate the diagnostic value of a combination of biomarkers to discriminate plaque ruptures in the setting of ACS. Eighty-five ACS patients with optical coherence tomography (OCT) images of the culprit plaque were included and categorized into two groups: ACS with plaque rupture (Rupture group, $n=42$ ) or without plaque rupture (Non-rupture group, $n=43$ ) verified by OCT. A discriminative model of plaque rupture using several biomarkers was developed and validated. The Rupture group had higher white blood cell (WBC) counts and peak creatine kinase-myocardial band (CK-MB) levels (13.39 vs. $2.69 \mathrm{ng} / \mathrm{mL}, \mathrm{p}=0.0016)$. sLOX-1 (227.9 vs. $51.7 \mathrm{pg} / \mathrm{mL}, \mathrm{p}<0.0001)$ and MMP-9 (13.4 vs. $6.45 \mathrm{ng} / \mathrm{mL}, \mathrm{p}=0.0313$ ) levels were significantly higher in the Rupture group, whereas NGAL showed a trend without statistical significance $(59.03 \mathrm{vs.} 53.80 \mathrm{ng} / \mathrm{mL}, \mathrm{p}=0.093$ ). Receiver operating characteristic curves to differentiate Rupture group from Non-rupture group calculated the area under the curve for sLOX-1 ( $p<0.001)$, MMP-9 $(p=0.0274)$, and NGAL $(p=0.0874)$ as $0.763,0.645$, and 0.609 , respectively. A new combinatorial discriminative model including sLOX-1, MMP-9, WBC count, and the peak CK-MB level showed an area under the curve of $0.8431(p<0.001)$. With a cut-off point of 0.614 , the sensitivity and specificity of plaque rupture were $62.2 \%$ and $97.6 \%$, respectively. The new discriminative model using sLOX-1, MMP-9, WBC count, and peak CK-MB levels could better identify plaque rupture than each individual biomarker in ACS patients.

Acute coronary syndrome (ACS) is a major concern for morbidity and mortality in patients suffered by ischemic heart disease $\mathrm{e}^{1,2}$. A complex and diverse process, including endothelial dysfunction, vascular inflammation, and hypercoagulability, result in atherosclerosis and plaque destabilization leading to $\mathrm{ACS}^{3,4}$. These pathophysiological components of coronary artery disease (CAD) may be detected by several biomarkers. Consequently, biomarker levels may be associated with the severity of CAD and hence may forecast the occurrence of adverse cardiovascular events or differentiate severe events from less severe ones in patients with CAD. Such associations have already been investigated for several biomarkers, such as C-reactive protein $(\mathrm{CRP})^{5-7}$, soluble lectin-like oxidized low-density lipoprotein (LDL) receptor-1 (sLOX-1), matrix metalloproteinase-9 (MMP-9), and neutrophil gelatinase-associated lipocalin (NGAL).

sLOX-1 is implied to be linked with vulnerable plaque and subsequent plaque rupture, and a previous study has reported that it may discriminate between ACS with and without plaque rupture ${ }^{8}$. Other studies have shown that MMP-9 indicates atherosclerotic plaque rupture or vulnerability by elastin degradation, which advances breakdown of the thin fibrous caps of plaques, ${ }^{90}$. Thus, MMP-9 activity is thought to be the key to ACS development. On the other hand, NGAL may be associated in the progression of atherosclerosis via endothelial dysfunction, inflammatory processes, and matrix degradation, leading to atherosclerotic plaque instability by modulating the activity of MMP-9 ${ }^{11-13}$. In the presence of CAD, plasma NGAL level is significantly elevated and

${ }^{1}$ Division of Cardiology, Department of Internal Medicine, Korea University Anam Hospital, Korea University School of Medicine, \#73, Inchon-ro, Seongbuk-gu, Seoul 02841, Korea. ${ }^{2}$ Department of Cardiovascular Medicine, Regional Cardiocerebrovascular Center, Wonkwang University Medical Center, Iksan, Korea. ${ }^{3}$ Health Insurance Review and Assessment Service, Wonju, Korea. ${ }^{\boxplus e m a i l}$ : ycw717@naver.com; dslmd@kumc.or.kr 
correlates with its severity and is notably higher in patients with acute myocardial infarction than in those with stable $\mathrm{CAD}^{13,14}$.

While the most common cause of intra-coronary thrombosis is plaque rupture, which found in approximately $50 \%$ of patients with $\mathrm{ACS}^{15-17}$, other causes include plaque erosion, calcified plaque, tight stenosis, intramural hematoma, and spontaneous dissection. Recent studies revealed that ACS patients presenting with plaque rupture have worse prognosis ${ }^{18,19}$. Therefore, differentiation between ruptured versus non-ruptured plaques in the culprit lesions is crucial for risk stratification and treatment strategy for patients with ACS.

Optical coherence tomography (OCT) has high resolution power and can discriminate several plaque characteristics of culprit lesions that result in $\mathrm{ACS}^{20-25}$. However, it is difficult to achieve timely risk stratification because OCT requires invasive and time-consuming coronary angiography.

Therefore, the identification of plaque ruptures without invasive testing in patients with ACS is necessary for quick risk assessment and appropriate management selection. Until now, data on the relationship between plaque rupture and serum biomarker levels are scarce.

In the present study, we sought to derive and validate a new discriminative model using several biomarkers for detecting plaque rupture in patients with ACS verified by OCT.

\section{Methods}

Study population and definitions. We screened 120 consecutive patients with ACS who arrived at the emergency room (ER) within $24 \mathrm{~h}$ after the onset of symptoms and underwent urgent percutaneous coronary intervention (PCI) between December 2014 and December 2015 at Korea University Anam Hospital. After the culprit lesions of ACS were identified based on coronary angiography findings, OCT examination of the culprit plaques was performed before PCI. Patients who did not undergo urgent PCI or those with cardiogenic shock, pulmonary congestion, fatal arrhythmia, or renal failure on regular hemodialysis were excluded from the study, as were those with inadequate OCT images due to massive residual thrombi or who required pre-dilation before OCT imaging ${ }^{8}$. As a result, 85 patients with appropriate OCT images were finally analyzed and categorized into two groups, namely, ACS with plaque rupture (Rupture group, $\mathrm{n}=42$ ) and ACS without plaque rupture (Nonrupture group, $\mathrm{n}=43$ ), verified by $\mathrm{OCT}^{8}$. The study protocol was approved by the Korea University Hospital Institute Review Board (2019AN0170), and written informed consent was obtained from all participants or their legal representatives. The study also complied with the Declaration of Helsinki.

ACS was defined as prolonged typical angina at rest $(\geq 20 \mathrm{~min})$ with significant coronary artery lesions confirmed on coronary angiography ${ }^{8}$. ST-segment elevation myocardial infarction (STEMI) was defined as ACS with new (or presumably new) ST-segment elevation $(\geq 0.1 \mathrm{mV})$ in two or more contiguous leads on electrocardiography and elevated cardiac troponin ${ }^{26}$. Non-STEMI (NSTEMI) was defined as ACS with new (or presumably new) ST-segment deviation $(\geq 0.05 \mathrm{mV})$ or T-wave inversion $(\geq 0.2 \mathrm{mV})$ in two or more contiguous leads on electrocardiography and elevated cardiac troponin ${ }^{8}$.

Blood sampling and measurement of biomarkers. Peripheral blood samples were collected from patients with ACS just before the PCI through the femoral or radial artery into a BD SST II Advance Tube (Becton, Dickinson and Company, Franklin Lakes, NJ, USA). Blood samples were allowed to clot for 30 min before $15 \mathrm{~min}$ of centrifugation at $1500 \times \mathrm{g}$. The serum was removed, aliquoted, and stored at $-80^{\circ} \mathrm{C}$ prior to the determination of serum sLOX-1, NGAL, and MMP-9 levels using enzyme-linked immunosorbent assay (ELISA). Serum sLOX-1 levels were assessed using the Human LOX-1 ELISA Kit (Cell Biolabs, Inc., San Diego, CA, USA). The lower limit of detection for sLOX-1 was $40 \mathrm{pg} / \mathrm{mL}^{27}$. Total human MMP-9 level was determined using the Quantikine Human MMP-9 Immunoassay (R\&D Systems, Inc., Minneapolis, MN, USA). The minimum detectable dose of MMP-9 was $0.156 \mathrm{ng} / \mathrm{mL}$. Intra- and inter-assay coefficients of variation for the MMP-9 immunoassay were $1.9-2.9 \%$ and $6.9-7.9 \%$, respectively. Serum NGAL levels were determined using the Quantikine Human Lipocalin-2/NGAL Immunoassay (R\&D Systems, Inc.). The minimum detectable dose of NGAL was $0.012 \mathrm{ng} / \mathrm{mL}$. The intra- and inter-assay coefficients of variation for the NGAL immunoassay were 3.1-4.4\% and 5.6-7.9\%, respectively. Serum high-sensitivity CRP levels were measured using latex-enhanced nephelometry (N-Latex CRP II; Siemens Healthcare Diagnostics, Tokyo, Japan). Serum high-sensitivity troponin I levels were measured using an electrochemiluminescence immunoassay (ECLusys Troponin hs; Roche Diagnostics, Tokyo, Japan). The assays were performed according to the manufacturer's instructions. Each serum sample was evaluated twice. ELISA-based measurements were obtained using the Versa Max Microplate Reader (Molecular Devices Corporation, Sunnyvale, California, USA) at an optical density of $450 \mathrm{~nm}$. Peripheral blood samples to measure serum creatine kinase-myocardial band (CK-MB) levels were serially obtained every $6 \mathrm{~h}$ after ER presentation until the CK-MB value began to decline, using an anti-human CK-MB monoclonal antibody (N-assay L CK-MB Nittobo; Nittobo Medical Co., Ltd., Fukushima, Japan).

OCT image acquisition and analysis. After aspiration of the thrombi using a thrombectomy catheter, whenever possible, the culprit lesion was examined using an OCT imaging catheter. OCT imaging of the culprit lesions (30 mm in length) was acquired with a frequency-domain OCT C7XR system and the Dragon Fly catheter (Lightlab Imaging/St. Jude Medical) ${ }^{8}$. In the C7XR system, a 2.7-F OCT imaging catheter was carefully advanced distal to the culprit lesion. The automated pullback was performed at $20 \mathrm{~mm} / \mathrm{s}$, while blood was displaced by a short injection of contrast media through the guiding catheter. The images were digitally stored for offline analysis. All OCT images were analyzed in the Korea University Anam Hospital core laboratory by two cardiologists (Jae-Young Cho and Hyung Joon Joo) who were blinded to the angiographic data and clinical presentations. When there was discordance between the observers, a consensus was obtained from an independent cardiologist. 


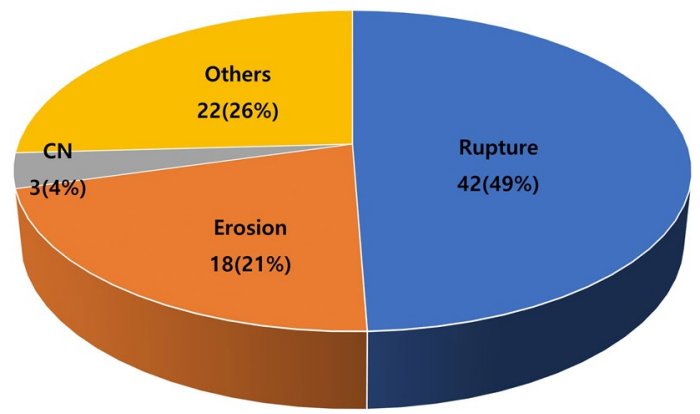

Figure 1. Composition of plaque morphology findings confirmed by OCT $(\mathrm{n}=85)$. $C N$ calcified nodule.

Definition of plaque morphology by OCT. Plaque rupture was defined as the presence of a fibrous cap discontinuity and cavity formation in the plaque ${ }^{28}$. Ruptured plaques usually have an extensive lipid core and a thin fibrous cap. The fibrous cap is the thinnest part at the rupture site, and the plaque cavity indicates the loss of lipid core due to rupture. Plaque erosion was defined as the presence of an irregular luminal surface without plaque rupture in OCT images ${ }^{28}$. A calcified nodule was defined as the protrusion of a signal-poor or heterogeneous region with a sharply delineated border. A thrombus was defined as a protrusion inside the lumen of the artery with signal attenuation. A white thrombus, which consists mainly of platelets, was identified as signal-rich, low-backscattering protrusions in the OCT image, while red thrombus was identified as highbackscattering protrusions inside the lumen of the artery with signal-free shadowing in the OCT image. Lipid cores were defined as diffusely bordered, signal-poor regions.

Quantitative coronary angiography. Coronary angiography was performed by engaging a Judkins catheter after puncturing the radial artery or femoral artery. Coronary angiograms were analyzed using the Cardiovascular Angiography Analysis System (Pie Medical Imaging B.V., Maastricht, the Netherlands). The reference diameter, minimum lumen diameter, diameter stenosis, area stenosis, and lesion length were measured with a computerized quantitative analyzer using a caliper.

Statistical analysis. Continuous variables are presented as mean \pm standard deviation and were compared using Student's t-test or Wilcoxon test and categorical variables using the chi-square test. Because sLOX-1, high-sensitivity CRP, troponin I, and CK-MB levels were not normally distributed, these values are presented as median and interquartile range (IQR) and were compared using the Wilcoxon test. Correlations between each biomarker level and the peak CK-MB level were analyzed using Spearman's rank order correlation test. The receiver operating characteristic (ROC) curve was used to assess whether the biomarkers (sLOX-1, NGAL, MMP-9) and white blood cell (WBC) count could differentiate between ruptured and non-ruptured plaques in patients with ACS. An area under the curve (AUC) of 1.0 indicated a test of perfect diagnostic value, whereas an AUC of 0.5 indicated no diagnostic value. The new discriminative model to detect plaque ruptures, which was built on multivariate logistic regression, was a score system ranging from 0 to 1 using a combination of sLOX-1, CK-MB, and MMP-9 levels and WBC count. The model equation formula is described below.

$$
\widehat{y}=\frac{\exp (-3.0137+0.00632 \times L O X-1+0.1843 \times W B C+0.00844 \times C K M B-0.0086 \times M M P-9)}{1+\exp (-3.0137+0.00632 \times \mathbf{L O X}-1+0.1843 \times \mathbf{W B C}+0.00844 \times \mathbf{C K M B}-0.0086 \times \mathbf{M M P}-9)}
$$

The best cut-off point was calculated using the Youden's index. When the model ROC curve was statistically significant, the curve was compared with the WBC count and sLox-1, NAGL, and MMP-9 levels. A two-sided p-value of $<0.05$ was considered statistically significant. Statistical analysis was performed using SAS 9.3 (SAS Institute Inc., Cary, NC, USA).

\section{Results}

OCT findings in patients with ACS. Plaque morphology findings confirmed by OCT in all 85 patients with ACS are shown in Fig. 1. Plaque rupture (49.4\%) was the most prevalent finding, followed by plaque erosion (21.2\%). The other findings included tight stenosis (22.4\%), calcified nodules (3.5\%), spontaneous dissection (2.4\%), and intramural hematoma (1.2\%).

Baseline characteristics. Comparisons of baseline characteristics between the Rupture group and Nonrupture group are summarized in Table 1. Patients in the Rupture group were more likely to be men (88.1 vs. $69.8 \%, \mathrm{p}=0.0387$ ) and have a clinical diagnosis of myocardial infarction (45.2 vs. $23.3 \%, \mathrm{p}=0.033)$ than those in the Non-rupture group. Ages were similar between the groups. Among the risk factors, body mass index (24.0, [22.9-26.7] vs. 23.4, [21.6-26.1] kg/m², $\mathrm{p}=0.0787)$ and incidence of diabetes mellitus (28.6 vs. $14.0 \%$, $\mathrm{p}=0.0991)$ showed a trend to be higher in the Rupture group but not hypertension, dyslipidemia, and smoking history. WBC count $\left(8.37,[7.04-11.46]\right.$ vs. $\left.6.82,[5.79-8.8] \times 10^{3} / \mu \mathrm{L}, \mathrm{p}=0.0098\right)$ was higher in the Rupture group, whereas lipid profiles showed no statistical differences between the groups. 


\begin{tabular}{|c|c|c|c|}
\hline & Rupture group $(n=42)$ & Non-rupture group $(n=43)$ & p-value \\
\hline Age, years & $60.2 \pm 11.5$ & $62.7 \pm 11.6$ & 0.3253 \\
\hline Male sex & $37(88.1)$ & $30(69.8)$ & 0.0387 \\
\hline BMI, kg/m² & $24.0(22.9-26.7)$ & $23.4(21.6-26.1)$ & 0.0787 \\
\hline \multicolumn{3}{|l|}{ Clinical diagnosis } & 0.0825 \\
\hline Unstable angina & $23(54.8)$ & $33(76.7)$ & \\
\hline NSTEMI & $7(16.7)$ & $5(11.6)$ & \\
\hline STEMI & $12(28.6)$ & $5(11.6)$ & \\
\hline Diabetes mellitus & $12(28.6)$ & $6(14.0)$ & 0.0991 \\
\hline Hypertension & $25(59.6)$ & $22(51.2)$ & 0.4382 \\
\hline Dyslipidemia & $41(97.6)$ & $43(100.0)$ & 0.3088 \\
\hline \multicolumn{3}{|l|}{ Smoking } & 0.1135 \\
\hline Smoker & $23(54.8)$ & $14(32.6)$ & \\
\hline Ex-smoker & $1(2.4)$ & $1(2.3)$ & \\
\hline Never smoker & $18(42.9)$ & $28(65.1)$ & \\
\hline Previous PCI & $8(19.1)$ & $7(16.3)$ & 0.7378 \\
\hline Previous CVA & $1(2.4)$ & $3(4.0)$ & 0.3171 \\
\hline Creatinine, mg/dL & $1.05(0.82-1.2)$ & $0.95(0.83-1.06)$ & 0.1112 \\
\hline Pro BNP, pg/mL & $158.5(44.1-709.6)$ & $81.3(19.5-610.3)$ & 0.4824 \\
\hline $\mathrm{WBC}, \times 10^{3} / \mu \mathrm{L}$ & $8.37(7.04-11.46)$ & $6.82(5.79-8.8)$ & 0.0098 \\
\hline Ejection fraction, \% & $60(50-60)$ & $60(57-60)$ & 0.1222 \\
\hline Total cholesterol, mg/dL & $172(59-213)$ & $168(130.5-201)$ & 0.8109 \\
\hline LDL-cholesterol, mg/dL & $113(101-159)$ & $111.5(96-151)$ & 0.5698 \\
\hline HDL-cholesterol, mg/dL & $44.5(35.5-83)$ & $49(41-62)$ & 0.4223 \\
\hline Triglyceride, mg/dL & $116(89-169)$ & $97(66-138)$ & 0.1403 \\
\hline
\end{tabular}

Table 1. Baseline characteristics. Data are presented as mean \pm SD or median (interquartile range) or $\mathrm{n}$ (percentage). BMI body mass index, NSTEMI non-ST-segment elevation myocardial infarction, STEMI ST-segment elevation myocardial infarction, PCI percutaneous coronary intervention, CVA cerebrovascular accidents, $B N P$ B-type natriuretic peptide, $W B C$ white blood cell, $L D L$ low-density lipoprotein, $H D L$ highdensity lipoprotein.

\begin{tabular}{|l|c|l|c|}
\hline & Rupture group $(\mathbf{n}=42)$ & Non-rupture group $(\mathbf{n}=43)$ & p-value \\
\hline Troponin I $(\mathrm{ng} / \mathrm{mL})$ & $0.21(0.12-5.82)$ & $0.19(0.1-0.92)$ & 0.7244 \\
\hline Peak CK-MB $(\mathrm{ng} / \mathrm{mL})$ & $13.39(2.23-182.8)$ & $2.69(1.65-5.19)$ & 0.0016 \\
\hline High-sensitivity CRP $(\mathrm{mg} / \mathrm{L})$ & $1.35(0.62-3.94)$ & $1.08(0.63-4.13)$ & 0.6635 \\
\hline sLOX-1 $(\mathrm{pg} / \mathrm{mL})$ & $227.87(49.45-607.3)$ & $51.7(19.6-104.3)$ & $<.0001$ \\
\hline NGAL $(\mathrm{ng} / \mathrm{mL})$ & $59.0(47.2-85.9)$ & $53.1(43.0-63.7)$ & 0.0932 \\
\hline MMP-9 $(\mathrm{ng} / \mathrm{mL})$ & $13.36(4.12-32.06)$ & $6.45(2.56-12.94)$ & 0.0313 \\
\hline
\end{tabular}

Table 2. Comparison of biomarker levels between Non-rupture group and Rupture group in patients with ACS. Data are presented as median (interquartile range). ACS acute coronary syndrome, $C K-M B$ creatine kinase-muscle/brain, $C R P$ C-reactive protein, $s L O X$-1 soluble lectin-like oxidized low-density lipoprotein receptor-1, NGAL neutrophil gelatinase-associated lipocalin, MMP-9 matrix metalloproteinase-9.

Comparison of biomarker levels between Rupture group and Non-rupture group. Different levels of various biomarkers within each group are shown in Table 2. Median values of the peak CK-MB (13.39 [2.23-182.8] vs. 2.69 [1.65-5.19] ng/mL, $\mathrm{p}=0.0016)$, sLOX-1 (227.87 [49.45-607.3] vs. 51.7 [19.6-104.3] pg/mL, $\mathrm{p}<0.0001)$, and MMP-9 (13.36 [4.12-32.06] vs. $6.45[2.56-12.94] \mathrm{ng} / \mathrm{mL}, \mathrm{p}=0.0313)$ levels were significantly higher in the Rupture group (Fig. 2). Scattered plot graph of peak CK-MB between two groups are presented in Supplementary Fig. S1. NGAL level $(59.0$ [47.2-85.9] vs. 53.1 [43.0-63.7] $\mathrm{ng} / \mathrm{mL}, \mathrm{p}=0.0932)$ was numerically higher in the Rupture group without statistical significance. Troponin I and high-sensitivity CRP levels were not different between the groups. This trend was maintained even when comparing Non-rupture group and Rupture group only in MI patients. Comparing biomarker levels between Non-rupture group and Rupture group in patients with MI are presented in Supplementary Table S1.

Correlation between peak CK-MB and biomarker levels. A correlation between the peak CK-MB and biomarker levels was observed (Fig. 3). sLOX-1 (Spearman $\rho=0.2821, \mathrm{p}=0.011$ ) and MMP-9 (Spearman 
sLOX-1

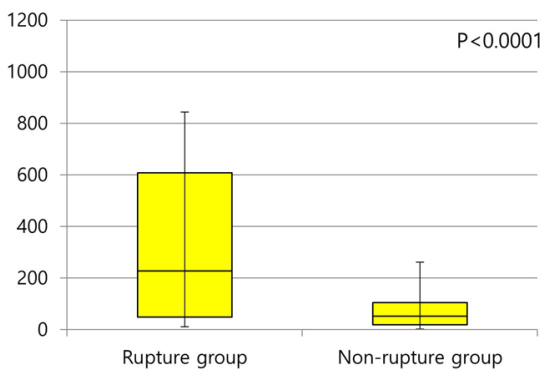

NGAL

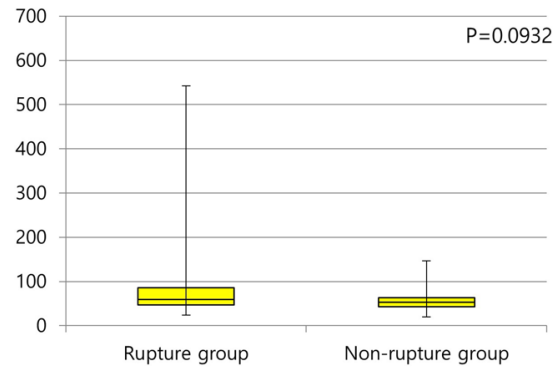

MMP-9

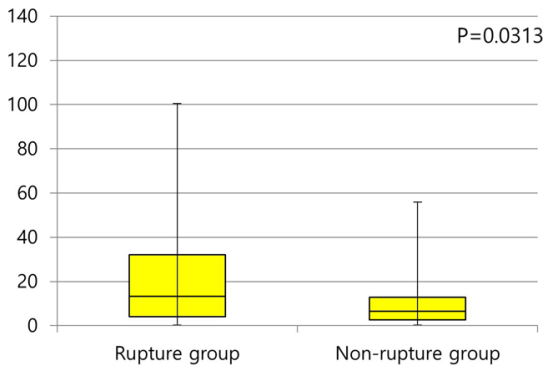

Figure 2. Comparison of sLOX-1, NGAL, and MMP-9 levels between the Rupture group and Non-rupture group. $s L O X-1$ soluble lectin-like oxidized low-density lipoprotein receptor-1, NGAL neutrophil gelatinaseassociated lipocalin, MMP-9 matrix metalloproteinase-9.
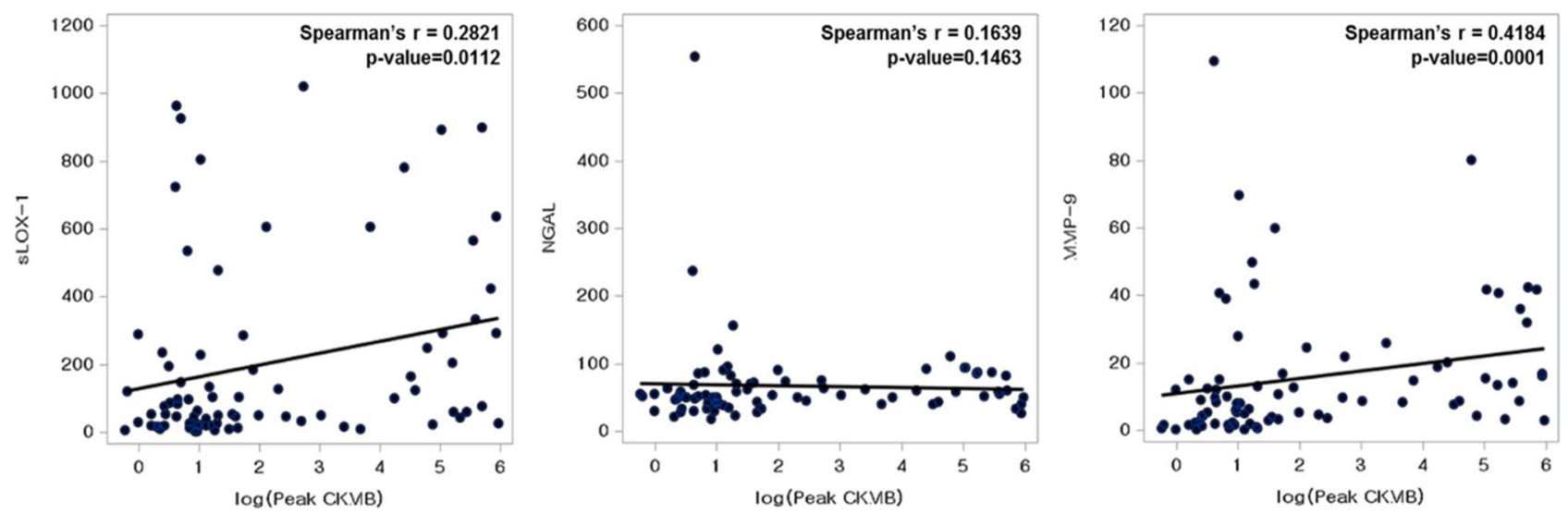

Figure 3. Correlation between the peak CK-MB level and biomarker levels. $C K-M B$ creatine kinase-muscle/ brain, $s L O X-1$ soluble lectin-like oxidized low-density lipoprotein receptor-1, NGAL neutrophil gelatinaseassociated lipocalin, MMP-9 matrix metalloproteinase-9.

$\rho=0.418, p=0.0001$ ) levels, but not NGAL (Spearman $\rho=0.164, p=0.146$ ) level, were significantly and proportionally correlated with the peak CK-MB level.

A new discriminative model using sLOX-1, MMP-9, and peak CK-MB and WBC to differentiate Rupture group from Non-rupture group. The ROC curve analysis for sLOX-1, NGAL, MMP-9, and WBC to detect Rupture group using Non-rupture group as a negative reference is shown in Fig. 4. The AUCs were $0.763(\mathrm{p}<0.0001), 0.645(\mathrm{p}=0.0274), 0.609(\mathrm{p}=0.0874), 0.665(\mathrm{p}=0.0016)$, and $0.701(\mathrm{p}=0.0007)$ for sLOX-1, MMP-9, NGAL, WBC, and the peak CK-MB level, respectively. The optimal cut-off values were $236.4 \mathrm{pg} / \mathrm{mL}$ (50\% sensitivity and $92.9 \%$ specificity), $12.7 \mathrm{ng} / \mathrm{mL}$ (54.8\% sensitivity and $74.4 \%$ specificity), $56.0 \mathrm{ng} / \mathrm{mL}$ ( $57.1 \%$ sensitivity and 65.1 specificity), and $6.900 / \mu \mathrm{L}$ (75.6\% sensitivity and $52.4 \%$ specificity) for sLOX-1, MMP-9, NGAL, and WBC count, respectively. The AUC value of the new discriminative model using LOX-1, MMP-9, WBC count, and the peak CK-MB level in combination was 0.843 ( $\mathrm{p}<0.0001)$, and the optimal cut-off value was 0.614 , which was calculated using the Youden's index $(62.2 \%$ sensitivity and $97.6 \%$ specificity; Fig. 5). The ROC curve of the new predictive model to detect Rupture group in patients with ACS was superior to $\operatorname{sLOX}-1(\mathrm{p}=0.0346)$, MMP-9 $(\mathrm{p}=0.0002)$, NAGL $(\mathrm{p}=0.0004)$, WBC count $(\mathrm{p}=0.0016)$, and peak CK-MB level alone $(\mathrm{p}=0.0199 ;$ Table 3$)$.

\section{Discussion}

Study findings. This study investigated the association between circulating plasma biomarkers and plaque characteristics defined by OCT in patients with ACS. Consequently, we demonstrated that plasma sLOX-1 and MMP-9 levels, but not NGAL levels, were higher in Rupture group than Non-rupture group. In addition, we evaluated a new discriminative model comprising several biomarkers, cardiac enzymes, and WBC count to differentiate Rupture group from Non-rupture group, which displayed an AUC of $0.843(\mathrm{p}<0.0001)$. When this model was compared with each biomarker, it showed an improved discriminatory ability to differentiate Rupture group from Non-rupture group.

The major findings of this study were as follows: (1) conventional cardiac biomarkers, including troponin I and high-sensitivity CRP, cannot differentiate between ACS with and without plaque ruptures, which is in line with previous findings ${ }^{8}$; (2) sLOX-1, MMP-9, and the peak CK-MB levels and WBC count have a potential to 

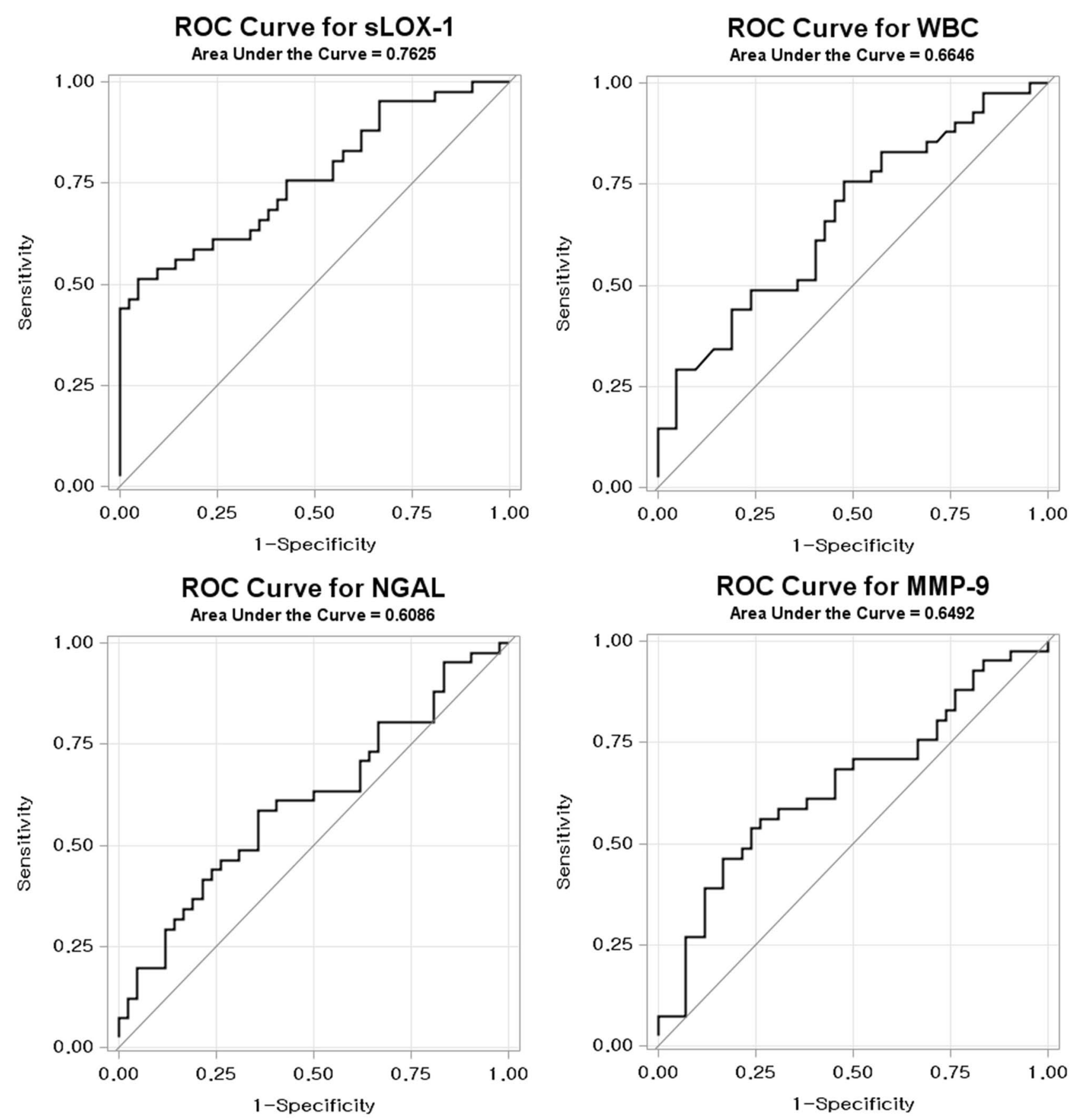

Figure 4. Receiver operating characteristic (ROC) curves for differentiating Rupture group from Non-rupture group. $s L O X-1$ soluble lectin-like oxidized low-density lipoprotein receptor-1, WBC white blood cell; NGAL neutrophil gelatinase-associated lipocalin, MMP-9 matrix metalloproteinase- 9 .

differentiate Rupture group from Non-rupture group, and among them, sLOX-1 has the greatest discriminatory ability; (3) a new discriminative model, developed by combining sLOX-1, MMP-9, and peak CK-MB levels with WBC count, could better discriminate plaque ruptures than individual biomarkers; (4) higher sLOX-1 and MMP-9 levels were well correlated with a higher peak CK-MB level and WBC count, which may reflect increased myocardial damage and subsequent inflammatory reaction in Rupture group than Non-rupture group; and (5) NGAL had no discriminatory power between Rupture group and Non-rupture group, although it is believed to be associated with poor clinical outcomes in $\mathrm{ACS}^{29}$.

Biomarkers and ACS. The peak CK-MB level represents the amount of myocardial damage in ACS, which is associated with worsened long-term prognosis. In the present study, sLOX-1 and MMP-9 were significantly correlated with the peak CK-MB level, whereas NGAL was not well correlated. 


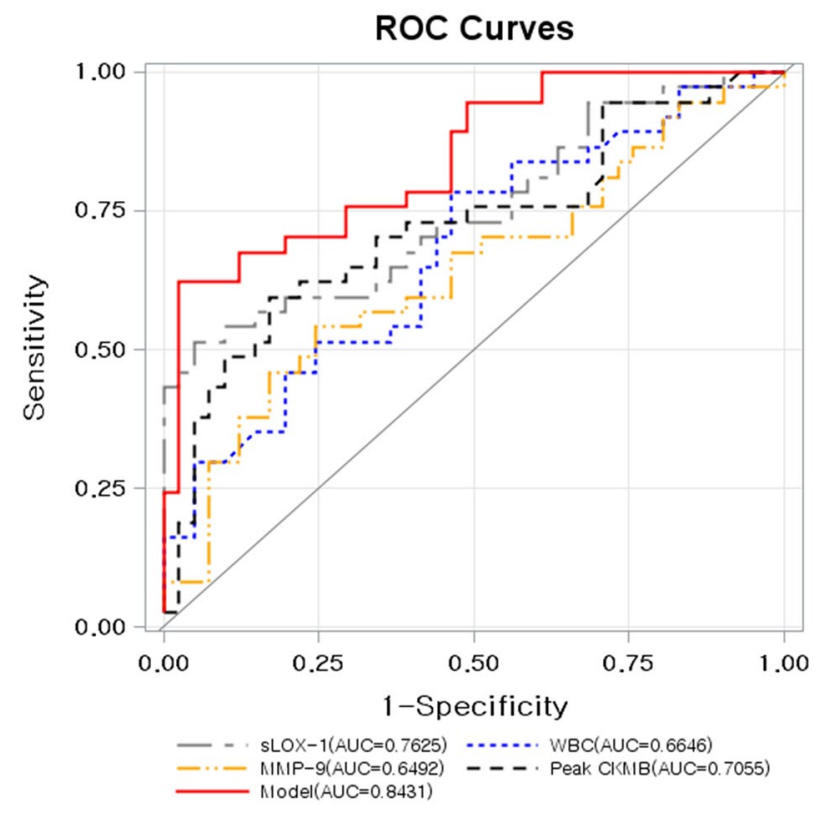

Figure 5. Receiver operating characteristics (ROC) curves displaying the additional discriminatory ability of the new predictive model using sLOX-1, MMP-9, and peak CK-MB levels and WBC count in combination for differentiating Rupture group from Non-rupture group. AUC area under the curve, $s L O X-1$ soluble lectin-like oxidized low-density lipoprotein receptor-1, $M M P-9$ matrix metalloproteinase-9, $C K-M B$ creatine kinasemuscle/brain; and WBC white blood cell.

\begin{tabular}{|l|l|l|l|l|l|}
\hline & AUC $(\mathbf{9 5} \% \mathbf{C I})$ & Cut-off point & Sensitivity & Specificity & p-value \\
\hline Model & $0.843(0.757,0.929)$ & 0.61 & 62.2 & 97.6 & - \\
\hline sLOX-1 & $0.763(0.660,0.865)$ & 236.4 & 50 & 95.35 & 0.0346 \\
\hline WBC & $0.665(0.548,0.781)$ & 6.9 & 75.61 & 52.38 & 0.0016 \\
\hline NGAL & $0.609(0.486,0.731)$ & 56.0 & 57.14 & 65.12 & 0.0004 \\
\hline MMP-9 & $0.645(0.524,0.766)$ & 12.7 & 54.76 & 74.42 & 0.0002 \\
\hline Peak CK-MB & $0.706(0.587,0.824)$ & 7.3 & 57.9 & 83.3 & 0.0199 \\
\hline
\end{tabular}

Table 3. Comparison between the new predictive model and each biomarker in terms of their power in differentiating Rupture group from Non-rupture group in patients with ACS. The cut-off point is calculated using the Youden's index. ACS acute coronary syndrome, $A U C$ area under the curve, $s L O X-1$ soluble lectin-like oxidized low-density lipoprotein receptor-1, WBC white blood cell, NGAL neutrophil gelatinase-associated lipocalin, $M M P-9$ matrix metalloproteinase- $9, C K-M B$ creatine kinase-muscle/brain. p-values are the comparison results between new predictive model and each biomarker.

MMPs, a family of zinc-containing endoproteinases, degrade the extracellular matrix components ${ }^{30}$. Vascular smooth muscle cells, macrophages, and endothelial cells express MMPs in accordance with inflammatory stimuli and oxidative stress. MMP-9 advances breakdown of the thin fibrous caps of plaques by elastic degradation, which indicates atherosclerotic plaque rupture or vulnerability ${ }^{9,10}$. A previous study has shown that patients with polymorphisms of the MMP-9 promotor have elevated MMP-9 expression, which was associated with acute myocardial infarction ${ }^{31}$. Many other studies have revealed that patients with ACS have significantly higher MMP-9 levels, with the highest in patients with STEMI, than in those with stable angina or healthy controls ${ }^{9,32-35}$.

However, Nishiguchi et al. reported that systemic MMP-9 level in patients with plaque rupture was equivalent to the level without plaque rupture ${ }^{36}$. In their study, local MMP-9 was higher in the plaque rupture group. Since the concentration of systemic MMP-9 was much dilute and lower than that of local MMP-9, more samples might have been needed to show statistical differences at lower concentrations. Also, there was a difference in the time taken for sampling between the present study and one by Nishiguchi et al., which may have influenced the results.

Several other studies have reported robust associations between MMP-9 level and the subsequent risk of cardiovascular complications, such as cardiovascular mortality and nonfatal myocardial infarction. Therefore, MMP-9 may act as not only a causative agent on plaque destabilization but also a circulating marker reflecting a proinflammatory state $\mathrm{s}^{37,38}$. 
Plasma oxidized LDL cholesterol levels are related to the thrombogenicity of coronary lesions in patients with unstable angina ${ }^{39}$. LOX-1, a scavenger receptor for the uptake of atherogenic oxidized LDL in the arterial wall, is plentifully expressed in advanced atherosclerotic plaque and plays an important role in the development of oxidative stress and inflammation ${ }^{40,41}$. When LOX-1 is cleaved by proteases at its proximal membrane extracellular domain following acute myocardial ischemia, sLOX-1 is released into the circulation ${ }^{42,43}$. sLOX-1 may arise from activated platelets, atheroma, or endothelial cells ${ }^{40}$. Therefore, circulating sLOX-1 levels are increased in patients with ACS. As a result, plasma sLOX-1 levels are a useful biomarker of ACS $^{44}$.

Taken together, MMP-9 is a causal proteinase that may contribute to plaque rupture by eroding or weakening a plaque cap and sLOX-1 is a consequent product of plaque rupture; thus, the two biomarkers directly reflect plaque rupture, and of the two, sLOX-1 has a stronger correlation with plaque rupture than MMP-9 in our study.

NGAL, a $25 \mathrm{kDa}$ glycoprotein isolated from the granule of mature neutrophils, covalently binds to MMP-9 and increases MMP-9 activity ${ }^{45}$, ${ }^{46}$. NGAL is expressed in smooth muscle cells, macrophages, and endothelial cells in atherosclerotic plaques ${ }^{11,47}$. Several studies have shown increased NGAL levels in CAD, which imply the involvement of NGAL in the atherosclerotic process ${ }^{14,48}$. In addition, a study has shown that NGAL levels were positively correlated with lesion complexity and the severity of CAD in patients with ACS ${ }^{49}$. However, NGAL binding to MMP-9 increases the rate of MMP-9/tissue inhibitor of metalloproteinase ${ }^{46}$. This stromal factor, the tissue inhibitor of metalloproteinase, inhibits the activity of MMP-9. Thus, NGAL activation plays as a modulator and leads to increased MMP-9 activity and plaque instability rather than plaque rupture itself. One study reported that NGAL is associated with long-term prognosis in patients with acute myocardial infarction because it has no direct relevance with plaque rupture but with plaque destabilization ${ }^{29}$.

Clinical implications of the identification of plaque rupture in patients with ACS. Several studies have proved that plaque rupture is the main cause of ACS and worsens the prognosis compared to Non-rupture group ${ }^{19,50,51}$. Plaque rupture is indeed the most common cause of coronary thrombosis, found in nearly $50 \%$ of patients with ACS, while plaque erosion is found in up to one-third of patients with ACS ${ }^{15-17}$. The other underlying mechanisms of ACS include calcified plaque, tight stenosis, intramural hematoma, and spontaneous dissection. Plaque rupture is often associated with positive remodeling, large plaque burden, and red thrombus ${ }^{52}$. Large plaque burdens in patients with plaque rupture may lead to residual plaque after PCI, which is a known predictor of stent failure ${ }^{19}$. Also, a previous study reported that patients with plaque rupture may have diffuse vulnerable features throughout the whole coronary tree ${ }^{18}$. These mechanisms together may reflect the worsened prognoses in patients with plaque rupture. On the other hand, plaque erosion is associated with negative remodeling, modest plaque burden, white thrombus, uncommon features of a fibroatheroma, and proximal distribution ${ }^{52}$. Given these findings, it may be reasonable to treat patients with plaque erosion by effective antithrombotic treatment without early invasive stent implantation ${ }^{53}$.

However, no single biomarker can successfully differentiate plaque rupture from non-plaque rupture in patients with ACS. Intravascular imaging tools such as OCT have advantages in detecting plaque rupture in the catheterization laboratory setting but have limitations in their usage due to the requirement of invasive procedures, such as coronary angiogram, low accessibility, high cost, possible complication risks, and time consumption. Therefore, discriminating ruptured and non-ruptured plaques using novel models comprising biomarkers without invasive procedures would be valuable for rapid risk stratification and predicting the prognoses of patients with ACS. At present, since new biomarkers including sLOX-1, MMP-9 are not established as routine laboratory tests, it may take considerable time to calculate this novel discriminative model and apply it to the treatment strategy of ACS patients. In our study, as described in the Methods section, it took about an hour to obtain each biomarker value after blood sampling. Calculating model and decision-making process would be faster if biomarker values are obtained though vein sampling as soon as patients with suspected ACS arrives at the emergency room. However, assumption of that values of biomarkers are similar between arterial sampling and venous sampling should be confirmed in future studies. With future technical advances to make inflammatory marker testing easier, more accurate and faster than it is today, we expect that the model developed in this study can be useful for identifying plaque ruptures quickly in bed-side setting and establishing treatment strategy accordingly. By quickly suspecting plaque rupture in an ACS setting using this discriminative model, the decision-making process for early invasive treatment strategies might be initiated earlier and more aggressive approach to use intravascular imaging tool to get stent optimization would be recommended, thereby improving the likelihood of better clinical outcomes. We believe that the present study is meaningful as it shows the possibility to create a more powerful model that can identify plaque rupture in patients with ACS using the existing biomarkers and laboratory data without invasive imaging tools.

Limitations. This study has several limitations. First, this study has all inherent limitations of a small-sized observational study. Second, we excluded patients requiring pre-dilation before OCT imaging. Third, because OCT signals may be interfered with by residual microthrombi, patients in the Non-rupture group may have had minor plaque rupture that was undetectable by OCT. Fourth, risk factors including sex, diabetes mellitus, and body mass index, which were different in both groups, may have affected the results of biomarkers. Fifth, although most sampling was performed just before the PCI procedure, the exact timing of blood sampling was not specified. Therefore, the effect of ischemic time on blood concentrations of biomarker could not be corrected because the time from symptom onset to sampling could not be accurately determined. However, because this study was conducted at a single institution with similar practices in treating patients with ACS and there was no difference in the ischemic time between the groups, the time from ischemic onset to sampling was likely to be similar in both groups. Finally, because blood sampling was performed through the femoral or radial artery rather than the coronary artery, blood concentrations of these biomarkers may have been affected by vascular 
beds other than the coronary artery. Therefore, sampling through the coronary artery is necessary to avoid this confounding factor in future research.

In conclusion, the new discriminative model using sLOX-1, MMP-9, and peak CK-MB levels and WBC count may identify plaque ruptures in an ACS setting. Further larger-sized prospective studies to externally validate the findings of this study are warranted.

Received: 31 December 2019; Accepted: 26 October 2020

Published online: 19 November 2020

\section{References}

1. Mozaffarian, D. et al. Executive summary: heart disease and stroke statistics-2016 update: a report from the American Heart Association. Circulation 133, 447-454. https://doi.org/10.1161/CIR.0000000000000366 (2016).

2. Mandelzweig, L. et al. The second Euro Heart Survey on acute coronary syndromes: characteristics, treatment, and outcome of patients with ACS in Europe and the Mediterranean Basin in 2004. Eur. Heart J. 27, 2285-2293. https://doi.org/10.1093/eurheartj/ ehl196 (2006).

3. Libby, P., Ridker, P. M. \& Maseri, A. Inflammation and atherosclerosis. Circulation 105, 1135-1143 (2002).

4. Hansson, G. K. Inflammation, atherosclerosis, and coronary artery disease. N. Engl. J. Med. 352, 1685-1695. https://doi.org/10.1056/ NEJMra043430 (2005).

5. James, S. K. et al. Troponin and C-reactive protein have different relations to subsequent mortality and myocardial infarction after acute coronary syndrome: a GUSTO-IV substudy. J. Am. Coll. Cardiol. 41, 916-924 (2003).

6. Makrygiannis, S. S. et al. Prognostic usefulness of serial C-reactive protein measurements in ST-elevation acute myocardial infarction. Am. J. Cardiol. 111, 26-30. https://doi.org/10.1016/j.amjcard.2012.08.041 (2013).

7. Sabatine, M. S. et al. Prognostic significance of the Centers for Disease Control/American Heart Association high-sensitivity $\mathrm{C}$-reactive protein cut points for cardiovascular and other outcomes in patients with stable coronary artery disease. Circulation 115, 1528-1536. https://doi.org/10.1161/CIRCULATIONAHA.106.649939 (2007).

8. Kobayashi, N. et al. Soluble lectin-like oxidized LDL receptor-1 (sLOX-1) as a valuable diagnostic marker for rupture of thincap fibroatheroma: verification by optical coherence tomography. Int. J. Cardiol. 168, 3217-3223. https://doi.org/10.1016/j.ijcar d.2013.04.110 (2013).

9. Kai, H. et al. Peripheral blood levels of matrix metalloproteases- 2 and -9 are elevated in patients with acute coronary syndromes. J. Am. Coll. Cardiol. 32, 368-372 (1998).

10. Loftus, I. M. et al. Increased matrix metalloproteinase- 9 activity in unstable carotid plaques. A potential role in acute plaque disruption. Stroke 31, 40-47 (2000).

11. Hemdahl, A. L. et al. Expression of neutrophil gelatinase-associated lipocalin in atherosclerosis and myocardial infarction. Arterioscler. Thromb. Vasc. Biol. 26, 136-142. https://doi.org/10.1161/01.ATV.0000193567.88685.f4 (2006).

12. $\mathrm{Bu}, \mathrm{D}$. X. et al. Induction of neutrophil gelatinase-associated lipocalin in vascular injury via activation of nuclear factor-kappaB. Am. J. Pathol. 169, 2245-2253 (2006).

13. Gupta, K., Shukla, M., Cowland, J. B., Malemud, C. J. \& Haqqi, T. M. Neutrophil gelatinase-associated lipocalin is expressed in osteoarthritis and forms a complex with matrix metalloproteinase 9. Arthritis Rheum. 56, 3326-3335. https://doi.org/10.1002/

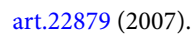

14. Zografos, T. et al. Association of neutrophil gelatinase-associated lipocalin with the severity of coronary artery disease. Am. J. Cardiol. 104, 917-920. https://doi.org/10.1016/j.amjcard.2009.05.023 (2009).

15. Cheruvu, P. K. et al. Frequency and distribution of thin-cap fibroatheroma and ruptured plaques in human coronary arteries: a pathologic study. J. Am. Coll. Cardiol. 50, 940-949. https://doi.org/10.1016/j.jacc.2007.04.086 (2007).

16. Virmani, R., Kolodgie, F. D., Burke, A. P., Farb, A. \& Schwartz, S. M. Lessons from sudden coronary death: a comprehensive morphological classification scheme for atherosclerotic lesions. Arterioscler. Thromb. Vasc. Biol. 20, 1262-1275 (2000).

17. Arbustini, E. et al. Plaque erosion is a major substrate for coronary thrombosis in acute myocardial infarction. Heart 82, 269-272 (1999).

18. Vergallo, R. et al. Pancoronary plaque vulnerability in patients with acute coronary syndrome and ruptured culprit plaque: a 3-vessel optical coherence tomography study. Am. Heart J. 167, 59-67. https://doi.org/10.1016/j.ahj.2013.10.011 (2014).

19. Niccoli, G. et al. Plaque rupture and intact fibrous cap assessed by optical coherence tomography portend different outcomes in patients with acute coronary syndrome. Eur. Heart J. 36, 1377-1384. https://doi.org/10.1093/eurheartj/ehv029 (2015).

20. Jang, I. K. et al. In vivo characterization of coronary atherosclerotic plaque by use of optical coherence tomography. Circulation 111, 1551-1555. https://doi.org/10.1161/01.CIR.0000159354.43778.69 (2005).

21. Kubo, T. et al. Assessment of culprit lesion morphology in acute myocardial infarction: ability of optical coherence tomography compared with intravascular ultrasound and coronary angioscopy. J. Am. Coll. Cardiol. 50, 933-939. https://doi.org/10.1016/j. jacc.2007.04.082 (2007).

22. Kubo, T. \& Akasaka, T. Optical coherence tomography imaging: current status and future perspectives: current and future developments in OCT. Cardiovasc. Interv. Ther. 25, 2-10. https://doi.org/10.1007/s12928-009-0006-3 (2010).

23. Bezerra, H. G., Costa, M. A., Guagliumi, G., Rollins, A. M. \& Simon, D. I. Intracoronary optical coherence tomography: a comprehensive review clinical and research applications. JACC Cardiovasc. Interv. 2, 1035-1046. https://doi.org/10.1016/j.jcin.2009.06.019 (2009).

24. Tanaka, A. et al. Morphology of exertion-triggered plaque rupture in patients with acute coronary syndrome: an optical coherence tomography study. Circulation 118, 2368-2373. https://doi.org/10.1161/CIRCULATIONAHA.108.782540 (2008).

25. Kubo, T. et al. Comparison of vascular response after sirolimus-eluting stent implantation between patients with unstable and stable angina pectoris: a serial optical coherence tomography study. JACC Cardiovasc. Imaging 1, 475-484. https://doi.org/10.1016/j. jcmg.2008.03.012 (2008).

26. Ibanez, B. et al. ESC Guidelines for the management of acute myocardial infarction in patients presenting with ST-segment elevation: the Task Force for the management of acute myocardial infarction in patients presenting with ST-segment elevation of the European Society of Cardiology (ESC). Eur. Heart J. https://doi.org/10.1093/eurheartj/ehx393 (2017).

27. Nakamura, M. et al. Generation of monoclonal antibodies against a soluble form of lectin-like oxidized low-density lipoprotein receptor- 1 and development of a sensitive chemiluminescent enzyme immunoassay. J. Pharm. Biomed. Anal. 51, 158-163. https ://doi.org/10.1016/j.jpba.2009.06.019 (2010).

28. Tearney, G. J. et al. Consensus standards for acquisition, measurement, and reporting of intravascular optical coherence tomography studies: a report from the International Working Group for intravascular optical coherence tomography standardization and validation. J. Am. Coll. Cardiol. 59, 1058-1072. https://doi.org/10.1016/j.jacc.2011.09.079 (2012). 
29. Lindberg, S. et al. Prognostic utility of neutrophil gelatinase-associated lipocalin in predicting mortality and cardiovascular events in patients with ST-segment elevation myocardial infarction treated with primary percutaneous coronary intervention. J. Am. Coll. Cardiol. 60, 339-345. https://doi.org/10.1016/j.jacc.2012.04.017 (2012).

30. Dollery, C. M., McEwan, J. R. \& Henney, A. M. Matrix metalloproteinases and cardiovascular disease. Circ. Res. 77, 863-868 (1995).

31. Kim, P. J. et al. Functional polymorphism in the promoter region of matrix metalloproteinase-9 is strongly associated with acute myocardial infarction. Korean Circ. J. 35, 192-196 (2005).

32. Hamed, G. M. \& Fattah, M. F. Clinical relevance of matrix metalloproteinase 9 in patients with acute coronary syndrome. Clin. Appl. Thromb. Hemost. 21, 705-711. https://doi.org/10.1177/1076029614567309 (2015).

33. Tanindi, A., Sahinarslan, A., Elbeg, S. \& Cemri, M. Relationship between MMP-1, MMP-9, TIMP-1, IL-6 and risk factors, clinical presentation, extent and severity of atherosclerotic coronary artery disease. Open Cardiovasc. Med. J. 5, 110-116. https://doi. org/10.2174/1874192401105010110 (2011).

34. Guo, C. et al. Correlation between the severity of coronary artery lesions and levels of estrogen, hs-CRP and MMP-9. Exp. Ther. Med. 7, 1177-1180. https://doi.org/10.3892/etm.2014.1565 (2014).

35. Nurkic, J., Ljuca, F., Nurkic, M., Jahic, E. \& Jahic, M. Biomarkers of plaque instability in acute coronary syndrome patients. Med. Arh. 64, 103-106 (2010).

36. Nishiguchi, T. et al. Local matrix metalloproteinase 9 level determines early clinical presentation of ST-segment-elevation myocardial infarction. Arterioscler. Thromb. Vasc. Biol. 36, 2460-2467. https://doi.org/10.1161/ATVBAHA.116.308099 (2016).

37. Blankenberg, S. et al. Plasma concentrations and genetic variation of matrix metalloproteinase 9 and prognosis of patients with cardiovascular disease. Circulation 107, 1579-1585. https://doi.org/10.1161/01.CIR.0000058700.41738.12 (2003).

38. Jonsson, S. et al. Increased levels of leukocyte-derived MMP-9 in patients with stable angina pectoris. PLoS ONE 6, e19340. https ://doi.org/10.1371/journal.pone.0019340 (2011).

39. Yamashita, H. et al. Elevated plasma levels of oxidized low-density lipoprotein relate to the presence of angiographically detected complex and thrombotic coronary artery lesion morphology in patients with unstable angina. Circ. J. 71, 681-687 (2007).

40. Pothineni, N. V. K. et al. LOX-1 in atherosclerosis and myocardial ischemia: biology, genetics, and modulation. J. Am. Coll. Cardiol. 69, 2759-2768. https://doi.org/10.1016/j.jacc.2017.04.010 (2017).

41. Sawamura, T. et al. An endothelial receptor for oxidized low-density lipoprotein. Nature 386, 73-77. https://doi.org/10.1038/38607 3a0 (1997).

42. Murase, T. et al. Identification of soluble forms of lectin-like oxidized LDL receptor-1. Arterioscler. Thromb. Vasc. Biol. 20, 715-720 (2000).

43. Mitsuoka, H. et al. Interleukin 18 stimulates release of soluble lectin-like oxidized LDL receptor-1 (sLOX-1). Atherosclerosis 202, 176-182. https://doi.org/10.1016/j.atherosclerosis.2008.04.002 (2009).

44. Hayashida, K. et al. Serum soluble lectin-like oxidized low-density lipoprotein receptor-1 levels are elevated in acute coronary syndrome: a novel marker for early diagnosis. Circulation 112, 812-818. https://doi.org/10.1161/CIRCULATIONAHA.104.46839 7 (2005).

45. Kjeldsen, L., Bainton, D. F., Sengelov, H. \& Borregaard, N. Identification of neutrophil gelatinase-associated lipocalin as a novel matrix protein of specific granules in human neutrophils. Blood 83, 799-807 (1994).

46. Yan, L., Borregaard, N., Kjeldsen, L. \& Moses, M. A. The high molecular weight urinary matrix metalloproteinase (MMP) activity is a complex of gelatinase B/MMP-9 and neutrophil gelatinase-associated lipocalin (NGAL). Modulation of MMP-9 activity by NGAL. J. Biol. Chem. 276, 37258-37265. https://doi.org/10.1074/jbc.M106089200 (2001).

47. Cruz, D. N., Gaiao, S., Maisel, A., Ronco, C. \& Devarajan, P. Neutrophil gelatinase-associated lipocalin as a biomarker of cardiovascular disease: a systematic review. Clin. Chem. Lab. Med. 50, 1533-1545. https://doi.org/10.1515/cclm-2012-0307 (2012).

48. Choi, K. M. et al. Implication of lipocalin-2 and visfatin levels in patients with coronary heart disease. Eur. J. Endocrinol. 158, 203-207. https://doi.org/10.1530/EJE-07-0633 (2008).

49. Soylu, K. et al. Serum neutrophil gelatinase-associated lipocalin levels are correlated with the complexity and the severity of atherosclerosis in acute coronary syndrome. Anatol. J. Cardiol. 15, 450-455. https://doi.org/10.5152/akd.2014.5513 (2015).

50. Davies, M. J. Anatomic features in victims of sudden coronary death. Coronary artery pathology. Circulation 85, 19-24 (1992).

51. Libby, P., Ridker, P. M. \& Hansson, G. K. Progress and challenges in translating the biology of atherosclerosis. Nature 473, 317-325. https://doi.org/10.1038/nature10146 (2011).

52. Kwon, J. E. et al. Multimodality intravascular imaging assessment of plaque erosion versus plaque rupture in patients with acute coronary syndrome. Korean Circ. J. 46, 499-506. https://doi.org/10.4070/kcj.2016.46.4.499 (2016).

53. Jia, H. et al. In vivo diagnosis of plaque erosion and calcified nodule in patients with acute coronary syndrome by intravascular optical coherence tomography. J. Am. Coll. Cardiol. 62, 1748-1758. https://doi.org/10.1016/j.jacc.2013.05.071 (2013).

\section{Acknowledgements}

The authors also would like to thank Yeunjin Park for her excellent assistance for English editing.

\section{Author contributions}

H.K. contributed to data analysis and interpretation and drafting the manuscript. D.H.J., J-Y.C., H.J.J., J.H.P. and S.J.H. contributed to data collection, interpretation and discussion of the study. J-H.K. contributed to the design concept. S.-A.C. contributed to the statistical analysis. D.-S.L. and C.W.Y. equally contributed to the study conception and design, data collection and interpretation, and revising and finalizing approval of the manuscript.

\section{Competing interests}

The authors declare no competing interests.

\section{Additional information}

Supplementary information is available for this paper at https://doi.org/10.1038/s41598-020-77413-3.

Correspondence and requests for materials should be addressed to C.W.Y. or D.-S.L.

Reprints and permissions information is available at www.nature.com/reprints.

Publisher's note Springer Nature remains neutral with regard to jurisdictional claims in published maps and institutional affiliations. 
(c) (i) Open Access This article is licensed under a Creative Commons Attribution 4.0 International cc) License, which permits use, sharing, adaptation, distribution and reproduction in any medium or format, as long as you give appropriate credit to the original author(s) and the source, provide a link to the Creative Commons licence, and indicate if changes were made. The images or other third party material in this article are included in the article's Creative Commons licence, unless indicated otherwise in a credit line to the material. If material is not included in the article's Creative Commons licence and your intended use is not permitted by statutory regulation or exceeds the permitted use, you will need to obtain permission directly from the copyright holder. To view a copy of this licence, visit http://creativecommons.org/licenses/by/4.0/.

(C) The Author(s) 2020 